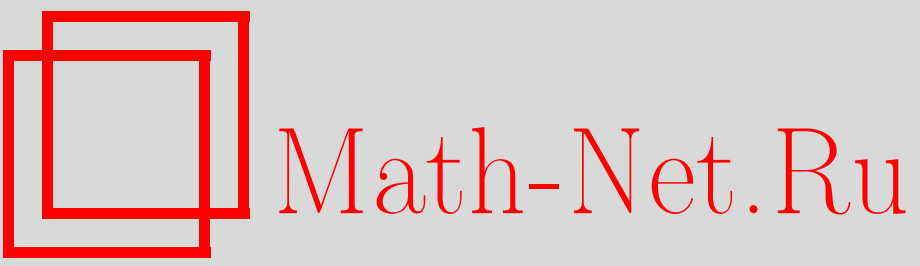

А. Векслер, Й. Зарми, Пертурбативный анализ взаимодействия волн в нелинейных системах, ТМФ, 2005, том 144, номер 2, 410-422

DOI: https://doi.org/10.4213/tmf1866

Использование Общероссийского математического портала Math-Net.Ru подразумевает, что вы прочитали и согласны с пользовательским соглашением http: //www. mathnet.ru/rus/agreement

Параметры загрузки:

IP : 54.81 .137 .203

26 апреля 2023 г., $17: 04: 53$ 


\section{ПЕРТУРБАТИВНЫЙ АНАЛИЗ ВЗАИМОДЕЙСТВИЯ ВОЛН В НЕЛИНЕЙНЫХ СИСТЕМАХ}

Предложен новый способ преодоления препятствий к асимптотической интегрируемости в возмущенных нелинейных дифференциальных уравнениях в частных производных в рамках метода нормальных форм (Н $\Phi)$ для случая многоволновых решений. Вместо того чтобы целиком включать препятствие в Н $\Phi$, туда включается только его резонансная часть (если таковая существует), а остаток относится к гомологическому уравнению. В результате НФ остается интегрируемой, а ее решения сохраняют характер решений невозмущенного уравнения. Произвол в разложении используется для построения канонических препятствий, которые ограничены областью взаимодействия волн. Для солитонных решений (например, в уравнении Кортевега-де Фриза) область взаимодействия является конечной областью вокруг начала координат; канонические препятствия при этом не порождают секулярных членов в гомологическом уравнении. Когда область взаимодействия является бесконечной (или полубесконечной - например, в решениях уравнения Бюргерса типа волновых фронтов), препятствия могут содержать резонансные члены. Препятствия порождают волны нового типа, которые нельзя записать в виде функционалов решений $\mathrm{H} \Phi$. Когда препятствие дает резонансный вклад в Н $\Phi$, происходит нестандартная корректировка волновой скорости.

Ключевые слова: нелинейные эволюционные уравнения, взаимодействия волн, препятствия к асимптотической интегрируемости, возмущенное уравнение Кортевега-де Фриза, возмущенное уравнение Бюргерса.

\section{1. ЗАДАЧА О ПРЕПЯТСТВИЯХ К АСИМПТОТИЧЕСКОЙ ИНТЕГРИРУЕМОСТИ}

Исследования эффектов возмушения волновых решений эволюционных дифференциальных уравнений в частных производных (ДУЧП) развивались в рамках двух различных подходов. В методе рассеяния решение невозмушенного уравнения рассеивается на возмушении, которое включается при $t=0$. Поскольку невозмушенное решение не является решением возмушенного уравнения, его амплитуда убывает и его волновое число,

\footnotetext{
* Department of Physics, Ben-Gurion University of the Negev, Beer-Sheva, 84105, Israel. E-mail: vekslera@bgu.ac.il, zarmi@bgu.ac.il

${ }^{\dagger}$ Department of Solar Energy \& Environmental Physics, Jacob Blaustein Institute for Desert Research, Sede-Boqer Campus, 84990, Israel
} 
скорость и фазовый сдвиг изменяются. Кроме того, в случае возмущенного уравнения Кортевега-де Фриза (КдФ) солитонный хвост был обнаружен вне солитонного сектора. Использованные методы представляли собой комбинацию метода обратной задачи рассеяния и процедуры множественного разложения по времени [1]-[8].

Второй подход состоит в другом. Интегрируемые нелинейные эволюционные уравнения являются приближением низшего порядка к более сложным уравнениям, описывающим полные динамические системы (например, уравнения динамики жидкости в случаях уравнений Бюргерса и КдФ и уравнения Максвелла в случае нелинейного уравнения Шредингера (НУШ)). Для улучшения аппроксимации эффекты высших порядков включаются в исходную физическую систему. При этом возмушение не включается при $t=0$. Оно сушествует во все моменты времени начиная с $t=-\infty$.

В этом подходе ишется решение нулевого порядка, которое имеет ту же волновую структуру, что и решение невозмушенного уравнения, за исключением изменения волновой скорости за счет эффектов высших порядков. Метод нормальных форм (НФ) применялся для анализа солитонных решений возмушенных уравнений КдФ и НУШ [9]-[13] и решений типа фронтов возмушенного уравнения Бюргерса [14]-[16]. Метод множественных временнь́хх масштабов использовался для возмушенного уравнения Кд $\Phi$ [17]-[19] и для возмушенного НУШ [20].

Мы сконцентрируем внимание на задачах, возникающих при анализе волновых решений в рамках второго подхода. Большинство интегрируемых нелинейных эволюционных ДУЧП допускают как одно-, так и многоволновые решения. Многоволновые решения, как правило, асимптотически стремятся к отчетливо разделенным одиночным волнам в $(x-t)$-плоскости, за исключением областей взаимодействия, где многоволновой характер решения теряется. Области взаимодействия могут быть локализованными (например, в случае многосолитонных решений уравнения КдФ) или полубесконечными (например, многофронтовые решения уравнения Бюргерса). Основная цель данной работы состоит в том, чтобы исследовать влияние, которое возмущения, добавляемые к невозмушенному уравнению, оказывают на волновые решения нелинейных систем.

Возмушенные уравнения часто анализируют в рамках метода НФ [14], [21], [22], который в кратком изложении состоит в следуюшем. Пусть

$$
w_{t}=F^{(0)}[w]+\sum_{k=1} \epsilon^{k} F^{(k)}[w]
$$

- возмушенное нелинейное эволюционное ДУЧП (квадратные скобки означают, что соответствуюший член является дифференциальным полиномом по $w(x, t))$. Мы предполагаем, что $w(x, t)$ можно разложить по малому параметру $\epsilon$ в степенной ряд дифференциальных многочленов от $u(x, t)$ (почти тождественное преобразование (ПТП)):

$$
w=\sum_{k=0} \epsilon^{k} u^{(k)}[u] \quad\left(u \equiv u^{(0)}\right) .
$$

Предполагается, что временна́я эволюция члена нулевого порядка $u(x, t)$ управляется $\mathrm{H} \Phi$ :

$$
u_{t}=\sum_{k=0} \epsilon^{k} \alpha_{k} S^{(k)}[u] \quad\left(a_{0}=1\right) .
$$


Здесь $S^{(k)}$ - резонансные члены, обычно называеммые симметриями. Их временна́я динамика эквивалентна динамике функции $u(x, t)$ с точностью до первого порядка, т.е.

$$
\left(u+\mu S^{(n)}\right)_{t}=F^{(0)}\left[u+\mu S^{(0)}\right] \quad(\mu \ll 1) .
$$

В результате их скобки Ли [23] обрашаются в нуль:

$$
\left[F^{(0)}, S^{(n)}\right] \equiv \sum_{i}\left\{\frac{\partial F^{(0)}}{\partial u_{i}} \partial_{x}^{i} S^{(n)}-\frac{\partial S^{(n)}}{\partial u_{i}} \partial_{x}^{i} F^{(0)}\right\}=0
$$

Симметрии (включая саму $F^{(0)}$ ) образуют иерархии [24], [25]. Можно установить $p e-$ куррентные соотношения между симметриями в каждой иерархии. Для многих уравнений (и всех уравнений, рассматриваемых в данной работе) первая симметрия имеет вид

$$
S^{(1)}[u]=u_{x}
$$

Подставляя ПТП (2) и НФ (3) в (1), получаем последовательностьгомологических уравнений для временно́й эволюции производных $u^{(n)}$, которую предстоит решить порядок за порядком.

Мотивировка предположения (3) состоит в том, что пертурбативный анализ, в котором резонансные члены не переносятся из гомологического уравнения в НФ, обычно приводит к секулярностям, т.е. неограниченным членам в приближенном решении. С другой стороны, от НФ ожидается интегрируемость и сохранение природы невозмущенного решения. Это свойство тесно связано с другим, весьма важным: при добавлении членов высших порядков к НФ основной эффект сводится к изменению физически значимых параметров (как правило, соотношения волновой скорости и дисперсии).

После устранения резонансных членов из гомологических уравнений они принимают вид

$$
\left[F^{(0)}, u^{(k)}[u]\right]+T^{(k)}[u]=0
$$

где $T^{(k)}[u]$ - вклад всех нерезонансных членов порядка $k$. ПТП строится из решений этих уравнений.

Однако начиная с некоторого порядка в разложении анализ может привести к выявлению препятствий к интегрируемости [9]-[13], [15]-[19], [26] - таких членов (дифференциальных полиномов), порождаемых в пертурбативном разложении динамического уравнения (1), которые нельзя учесть в формализме. Структура препятствий как дифференциальных полиномов не однозначна и зависит от способа, которым строится ПТП.

Для того чтобы сделать построение ПТП возможным, обычный способ действий состоит в добавлении этих неучтенных членов в НФ. Это делает НФ неинтегрируемой, откуда и происходит название "препятствие к интегрируемости". Включение препятствий в НФ возмушает волновой характер ее решений. В случае двухсолитонного решения возмушенного уравнения КдФ в НФ [9]-[11] эффект препятствий изучался в [13], [27]. Было найдено, что решению в нулевом порядке свойственны неупругие эффекты: появление излученной волны второго порядка, зависящие от времени поправки четвертого порядка к каждому из волновых чисел, а также генерация солитона восьмого порядка. 


\section{2. ПОДХОД К ПРЕОДОЛЕНИЮ ПРЕПЯТСТВИЙ}

2.1. Общие идеи. Необходимость включения препятствий в НФ следует из допущения, обычно принимаемого в НФ-разложении, что все члены в ПТП являются дифференциальными полиномами в приближении нулевого порядка (т.е. полиномами по $u(x, t))$ и не зависят явно от независимых переменных $t$ и $x$. В нашем подходе эта проблема преодолевается за счет того, что допускается зависимость от этих переменных членов высших порядков в ПТП. Для этого мы предполагаем следующий вид члена $k$-го порядка в ПТП:

$$
u^{(k)}=u_{d}^{(k)}[u]+u_{r}^{(k)}(x, t) .
$$

В уравнении (8) $u_{d}^{(k)}[u]$ - дифференциальный полином по $u$, а $u_{r}^{(k)}(x, t)$ явно зависит от $x$ и $t$ и служит, как предполагается, для учета препятствий. Таким образом, подставляя допущение (8) в гомологическое уравнение (7), получаем

$$
\left[F^{(0)}, u_{r}^{(k)}(x, t)\right]+R^{(k)}[u]=0
$$

где $R^{(k)}[u]$ обозначает препятствие порядка $k$.

Из-за произвола, присушего пертурбативному разложению, конструкция для $u_{d}^{(k)}[u]$ не является однозначной. За исключением случая, когда $u_{d}^{(k)}[u]$ выбрано подходящим образом, получающееся препятствие может не отражать следующие свойства, представляющие физический интерес:

а) препятствия не возникают в случае одноволновых решений НФ [13];

б) ожидание, что препятствия возникают из-за взаимодействия между волнами в многоволновом случае [12].

Оба свойства реализуются, если $u_{d}^{(k)}[u]$ выбрано так, чтобы оно имело структуру дифференциального полинома, который решает задачу в случае одноволнового решения НФ. Выбор, предложенный для $u_{d}^{(k)}[u]$, приводит препятствия к "каноническому" виду, выражаемому в терминах симметрий невозмущенного уравнения. Препятствия тог да обрашаются в нуль, если подставить для $u$ одноволновое решение НФ. Более важно, что в результате ожидается, что они обратятся в нуль за пределами областей взаимодействия волн в многоволновом случае. Причина состоит в том, что вне областей взаимодействия многоволновые решения асимптотически стремятся к сумме отчетливо разделенных одноволновых решений.

2.2. Конструкция канонических препятствий. Начав с $u_{d}^{(k)}[u]$ - решения гомологического уравнения (7) для одной волны, находим, что канонические препятствия можно записать в виде

$$
\begin{gathered}
R^{(n)}[u]=\sum_{\substack{k=3 \\
i+j=k}}^{n+g} \gamma_{k}^{n} f_{i j}\left[u, \partial_{x}\right] R_{i j}[u], \\
R_{p q}[u]=S^{(p)}[u] G^{(q)}[u]-S^{(q)}[u] G^{(p)}[u], \\
S^{(n)}[u]=\partial_{x} G^{(n)}[u],
\end{gathered}
$$


где $\gamma_{k}^{n}$ - численный коэффициент, $f_{i j}\left[u, \partial_{x}\right]$ - дифференциальный оператор подходяшего веса, а $g$ - интервал между индексами симметрии и порядком возмушения. Например, если определить $F^{(0)}$ как $S^{(2)}$ (что представляет собой широко распространенное обозначение), то $g=2$.

Препятствия уравнения (10) тождественно обращаются в нуль в случае одноволновых решений НФ. Чтобы увидеть это, используем тот факт, что все симметрии пропорциональны друг другу в случае одноволновых решений НФ. Для “тривиального" граничного условия $u(\xi \rightarrow-\infty)=0$ имеем

$$
S^{(n)}=(-1)^{n+1} v_{0}^{n+1} S^{(1)} .
$$

Это можно доказать по индукции для любой иерархии, которая подчинена линейному рекуррентному соотношению.

Пропорциональность всех симметрий приводит к простому изменению скорости решений НФ:

$$
v=\sum_{k \geqslant 0} \epsilon^{k} v_{k}, \quad v_{k}=(-1)^{k} a_{k} v_{0}^{k+1}
$$

Уравнение (13) также описывает изменение скорости каждой волны в многоволновом решении.

2.3. Резонансный вклад в препятствиях? Для многоволновых решений НФ препятствия не обращаются в нуль. Важная характеристика канонических препятствий состоит в том, что они не обращаются в нуль только в пределах областей взаимодействия на $(x-t)$-плоскости. Например, в случае $\mathrm{K}$ д является конечная область вокруг начала координат, тогда как в случае фронтов Бюргерса она состоит из одной или большего числа областей конечной ширины вдоль полубесконечных линий. Канонические препятствия обрашаются в нуль экспоненциально быстро за пределами областей взаимодействия, где решение асимптотически приближается к отчетливо разделенным отдельным волнам. Напротив, неканонические препятствия являются конечными также и вне области взаимодействия.

Наиболее существенный вопрос, который теперь возникает, состоит в том, порождают ли препятствия секулярные члены в ПТП (2). Симметрия, если таковая содержится в препятствии, порож дает секулярный член посредством уравнения (9). Мы предлагаем поэтому разбить препятствие в сумму симметрии и нерезонансного члена. Эта симметрия со своим коэффициентом должна быть включена в НФ (3).

Наша задача заключается в том, чтобы определить, способно ли каноническое препятствие породить секулярные члены. Простой критерий для выявления таковой способности состоит в том, что препятствие распространяется по бесконечной или полубесконечной области, асимптотически приближаясь к симметрии. (Этот критерий подобен альтернативе Фредгольма, хотя и является намного менее строгим.)

Мы остановимся подробнее на двухволновых решениях двух уравнений: двухсолитонном решении уравнения Кд фронтовом решении уравнения Бюргерса, где препятствие возникает уже в первом порядке. 
Для уравнения КдФ область взаимодействия и тем самым препятствия локализованы. Поэтому мы ожидаем, что решение уравнения (9) для этой задачи будет ограниченным. Это было проверено путем численного решения гомологического уравнения. Мы получаем новую ограниченную солитоноподобную волну.

С другой стороны, область взаимодействия в двухфоронтовом решении уравнения Бюргерса является полубесконечной (фронты отчетливо разделены в одной половине $(x-t)$-плоскости и сливаются (взаимодействуя) в другой половине). Каноническоепрепятствие асимптотически приближается к симметрии $S^{(3)}[u]$. Поэтому мы ожидаем, что препятствие Бюргерса породит секулярный член в уравнении (9). Это было проверено путем численного решения уравнения.

Мы "извлекаем" симметрию $S^{(3)}[u]$ из препятствия вместе с коэффициентом и пере-

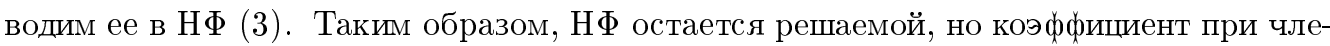
не первого порядка в измененной волновой скорости изменяется. Остаток не является каноническим препятствием, т.е. не ограничен областью взаимодействия, а напротив, распространяется по всем фронтам решения нулевого порядка. Однако он не содержит симметрию. Решение гомологического уравнения должно быть ограниченным. В замкнутом виде решений гомологического уравнения нет, но численный анализ показывает, что наше предсказание подтверждается.

В следуюшем разделе эти два примера рассмотрены достаточно подробно. Полные результаты нашей работы будут приведены в последуюшей публикации. Подчеркнем, что наши результаты критическим образом зависят от волновой природы решений.

2.4. Итоги и заключение. 1. Главный эффект влияния возмущений на взаимодействие волн в многоволновых решениях нелинейных ДУЧП состоит в появлении препятствий к интегрируемости.

2. После перенесения резонансной части препятствий в НФ адекватным способом работы с ними становится гомологическое уравнение. Для этого необходимо допустить явную зависимость членов высших порядков в ПТП (2) от независимых переменных. В действительности $u^{(n)}$ состоит из двух частей: дифференциального полинома $u_{d}^{(n)}[u]$, структура которого соответствует случаю одноволнового решения $\mathrm{H} \Phi$ (3) (причем препятствий тогда нет), и функции $u_{r}^{(n)}(x, t)$, которая должна учитывать препятствие.

3. Когда ПТП строится указанньм образом, препятствия (если они сушествуют) получаются в канонической форме (10). Канонические препятствия ограничены областью взаимодействия волн.

4. Когда область взаимодействия локализована (например, для КдФ-солитонов), канонические препятствия не порождают секулярных решений гомологических уравнений (9). Решение гомологического уравнения является тогда ограниченным, и, как правило, недоступно в замкнутом виде.

5. Когда область взаимодействия занимает полубесконечные пределы, ожидается, что препятствия приведут к появлению секулярных решений. Тогда требуется отождествить симметрию, "скрытую" внутри препятствия, и перенести ее из гомологического уравнения в $\mathrm{H} \Phi$. Остаток препятствия сохраняется в гомологическом уравнении и приводит к ограниченным решениям. 
В обеих ситуациях препятствия перестают быть препятствиями к интегрируемости НФ. Она остается интегрируемой, а ее решения (приближения нулевого порядка) сохраняют характер невозмушенных решений. Различие между приведенными вьше пунктами 4 и 5 состоит в том, что в пункте 5 затрагивается еще и волновая скорость, в том порядке, в котором сушествует препятствие. Сосредоточившись на двухволновых решениях, мы нашли, что препятствие, как правило, приводит к дополнительной волне, которая может не выражаться в замкнутом виде. В общем случае гомологическое уравнение следует решать численно.

\section{3. РЕШЕНИЕ ДВУХ ПРИМЕРОВ}

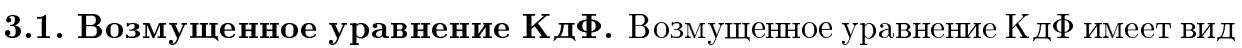

$$
\begin{aligned}
w_{t}= & 6 w w_{x}+w_{x x x}+\epsilon\left(30 \alpha_{1} w^{2} w_{x}+10 \alpha_{2} w w_{x x}+20 \alpha_{3} w_{x} w_{x x}+\alpha_{4} w_{5 x}\right)+ \\
& +\epsilon^{2}\left(140 \beta_{1} w^{3} w_{x}+70 \beta_{2} w^{2} w_{x x x}+280 \beta_{3} w w_{x} w_{x x}+14 \beta_{4} w w_{5 x}+\right. \\
& \left.+70 \beta_{5} w_{x}^{3}+42 \beta_{6} w_{x} w_{4 x}+70 \beta_{7} w_{x x} w_{x x x}+\beta_{8} w_{7 x}\right)+O\left(\epsilon^{3}\right) .
\end{aligned}
$$

Предположим, что ПТП имеет вид

$$
w=u+\epsilon u^{(1)}+\epsilon^{2} u^{(2)}+O\left(\epsilon^{3}\right)
$$

и НФ -

$$
u_{t}=S^{(2)}[u]+\epsilon \alpha_{4} S^{(3)}[u]+\epsilon^{2} \beta_{8} S^{(4)}[u]+O\left(\epsilon^{3}\right),
$$

где

$$
\begin{aligned}
S^{(2)}[u]= & 6 u u_{x}+u_{x x x} \\
S^{(3)}[u]= & 30 u^{2} u_{x}+10 u u_{x x}+20 u_{x} u_{x x}+u_{5 x} \\
S^{(4)}[u]= & 140 u^{3} u_{x}+70 u^{2} u_{x x}+280 u_{x} u_{x x}+14 u u_{5 x}+ \\
& +70 u_{x}^{3}+42 u_{x} u_{4 x}+70 u_{x x} u_{x x x}+u_{7 x} .
\end{aligned}
$$

Одноволновое решение НФ (16) является хорошо известным КдФ-солитоном:

$$
u(x, t)=\frac{2 k^{2}}{\operatorname{ch}^{2}\left[k\left(x-v t+x_{0}\right)\right]},
$$

а двухволновое решение дается формулой Хироты [28]

$$
\begin{gathered}
u(x, t)=2 \partial_{x}^{2} \ln \left\{1+g_{1}(x, t)+g_{2}(x, t)+\left(\frac{k_{1}-k_{2}}{k_{1}+k_{2}}\right) g_{1}(x, t) g_{2}(x, t)\right\}, \\
g_{i}(x, t)=\exp \left[2 k_{i}\left(x-v_{i} t+x_{0, i}\right)\right] .
\end{gathered}
$$

Пример этого решения представлен на рис. 1. Единственное различие между решениями невозмушенного уравнения $\mathrm{K}$ д $w_{t}=6 w w_{x}+w_{x x x}$ и обоими решениями Н $\Phi$ состоит в изменении волновой скорости:

$$
v_{i}=-4 k_{i}^{2}-16 \epsilon \alpha_{4} k_{i}^{4}-64 \epsilon^{2} \beta_{8} k_{i}^{6}-O\left(\epsilon^{3}\right) .
$$




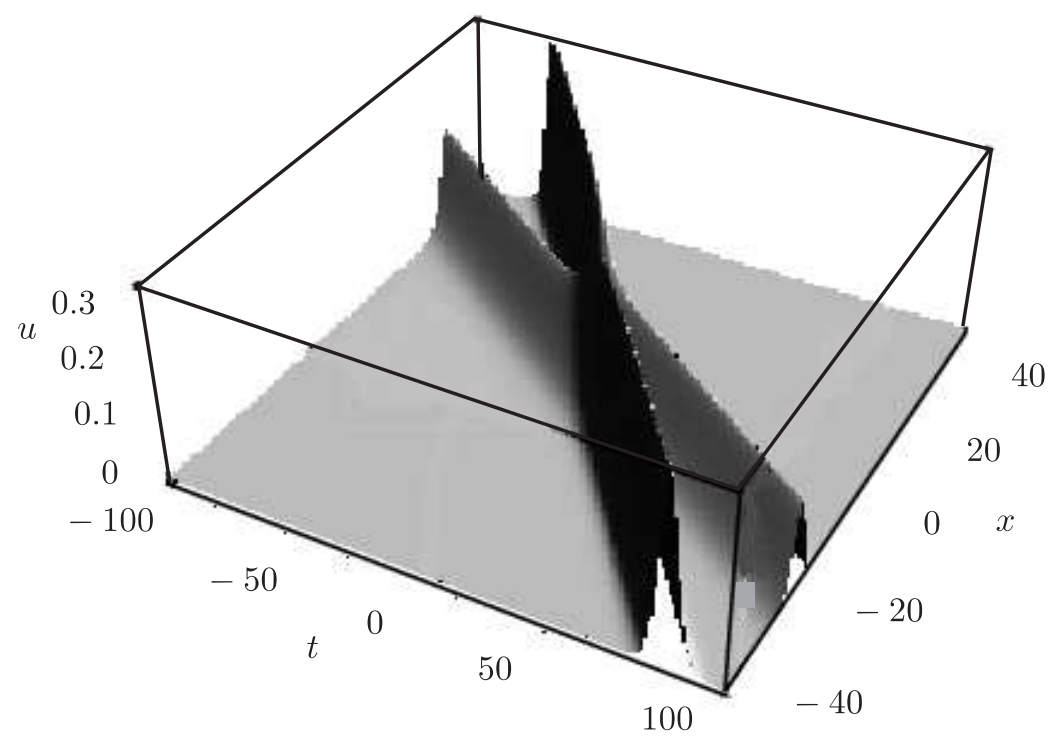

Рис. 1. Двухсолитонное решение Н $\Phi$ уравнения $\mathrm{K}$ д $\Phi$ (уравнение (19)); $k_{1}=0.3, k_{2}=$ 0.4 .

При анализе НФ не возникает препятствий в первом порядке. Однако препятствие появляется во втором порядке. Выбирая член второго порядка в ПТП в виде

$$
\begin{gathered}
u^{(2)}=u_{r}^{(2)}(x, t)+B_{1} u^{3}+B_{2} u u_{x x}+B_{3} u_{x}^{2}+B_{4} u_{x x x x}+ \\
+B_{5} u u_{x} q^{(1)}+B_{6} u_{x x x} q^{(1)}+B_{7} u_{x x} q^{(1)^{2}}+B_{8} u_{x} q^{(2)} \\
q^{(1)} \equiv \partial_{x}^{-1} u, \quad q^{(2)} \equiv \partial_{x}^{-1}\left(u^{2}\right)
\end{gathered}
$$

при подходящем наборе значений $\left\{B_{k}\right\}$, основанном на виде функции $u^{(2)}$ в случае односолитонного решения, получаем каноническое препятствие

$$
R^{(2)}=\gamma_{3}^{2} u R_{21}=\gamma_{3}^{2} u\left(3 u^{2} u_{x}+u u_{x x x}-u_{x} u_{x x}\right)
$$

$\left(\gamma_{3}^{2}\right.$ строится как комбинация коэффициентов в уравнении $\left.(14)\right)$. Это препятствие локализовано (см. рис. 2), а потому не может порождать секулярного решения в гомологическом уравнении

$$
\partial_{t} u_{r}^{(2)}=6 \partial_{x}\left(u u_{r}^{(2)}\right)+\partial_{x}^{3} u_{r}^{(2)}+\gamma_{3}^{2} u R_{21}
$$

Это уравнение можно решить в замкнутом виде методом функций Грина, развитым в контексте обратной задачи рассеяния [2], [29]-[31]. Поскольку наша цель состоит только в том, чтобы продемонстрировать решение уравнения (23), численного решения для этого вполне достаточно. Оно демонстрирует новую ограниченную солитоноподобную волну (рис. 3 ). 


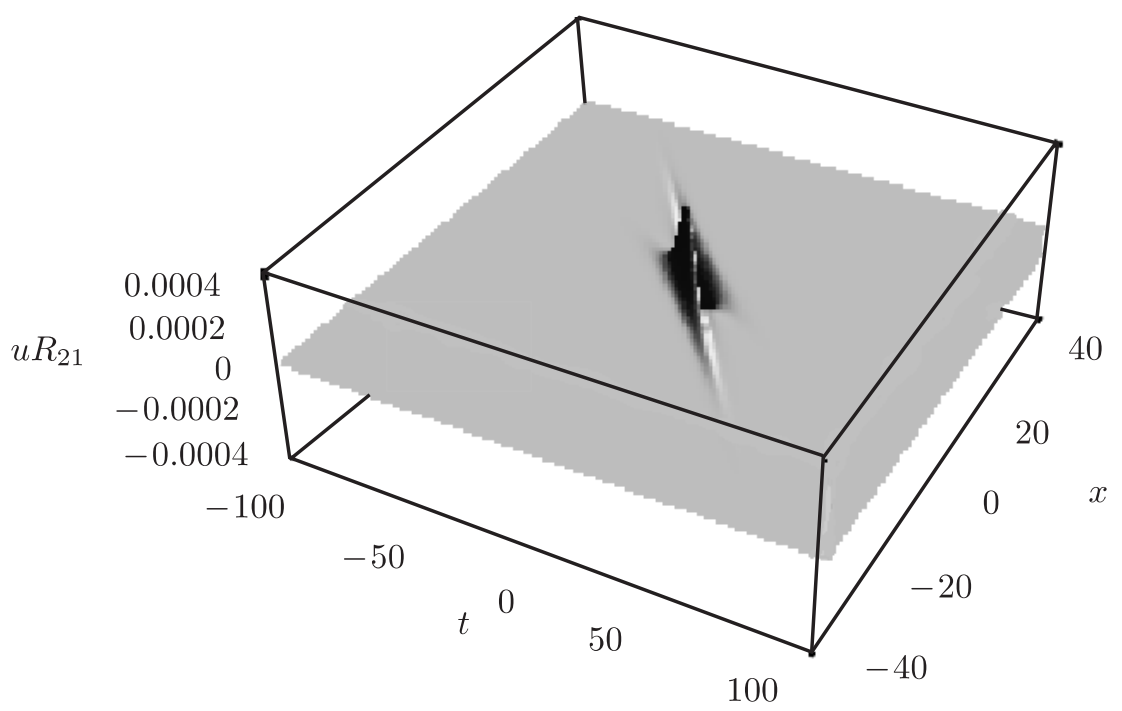

Рис. 2. Каноническое препятствие $u R_{21}$ для двухсолитонного решения уравнения Кд $\Phi$ (уравнение (22)); параметры те же, что и на рис. 1.

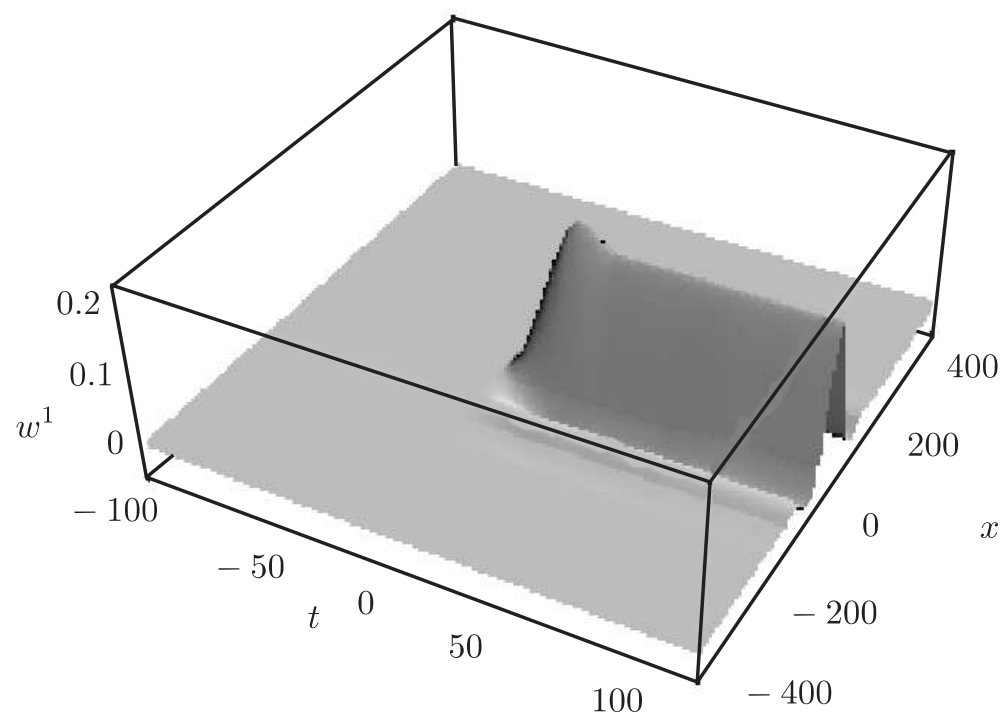

Рис. 3. Вклад канонического препятствия $u R_{21}$ в $u_{r}^{(2)}$ из уравнения (23) для нулевого граничного условия при $x \rightarrow-\infty ; k_{1}=0.5, k_{2}=0.75$.

3.2. Возмущенное уравнение Бюргерса. Возмущенное уравнение Бюргерса имеет вид

$$
w_{t}=2 w w_{x}+w_{x x}+\epsilon\left(3 \alpha_{1} w^{2} w_{x}+3 \alpha_{2} w w_{x x}+3 \alpha_{3} w_{x}^{2}+\alpha_{4} w_{x x x}\right)+O\left(\epsilon^{2}\right)
$$


(мы не пишем здесь возмушение второго порядка, поскольку препятствие возникает уже в первом порядке). Мы снова предполагаем, что ПТП имеет вид

$$
w=u+\epsilon u^{(1)}+O\left(\epsilon^{2}\right)
$$

и Н $\Phi-$

$$
u_{t}=2 u u_{x}+u_{x x}+\epsilon \mu\left(3 u^{2} u_{x}+3 u u_{x x}+3 u_{x}^{2}+u_{x x x}\right)+O\left(\epsilon^{2}\right) .
$$

Ее одноволновое решение представляет собой ударньй фронт

$$
u(x, t)=\frac{k A \exp [k(x-v t)]}{1+k A \exp [k(x-v t)]}
$$

а двухфронтовое решение дается непосредственным продолжением формулы (27):

$$
u(x, t)=\frac{k_{1} A_{1} \exp \left[k_{1}\left(x-v_{1} t\right)\right]+k_{2} A_{2} \exp \left[k_{2}\left(x-v_{2} t\right)\right]}{1+k_{1} A_{1} \exp \left[k_{1}\left(x-v_{1} t\right)\right]+k_{2} A_{2} \exp \left[k_{2}\left(x-v_{2} t\right)\right]}
$$

(пример его приведен на рис. 4 , причем можно видеть, что фронты взаимодействуют, т.е. сливаются, в полубесконечной области). Измененная скорость по-прежнему одинакова для обоих решений:

$$
v_{i}=-k_{i}-\epsilon \mu k_{i}^{2}-O\left(\epsilon^{2}\right)
$$

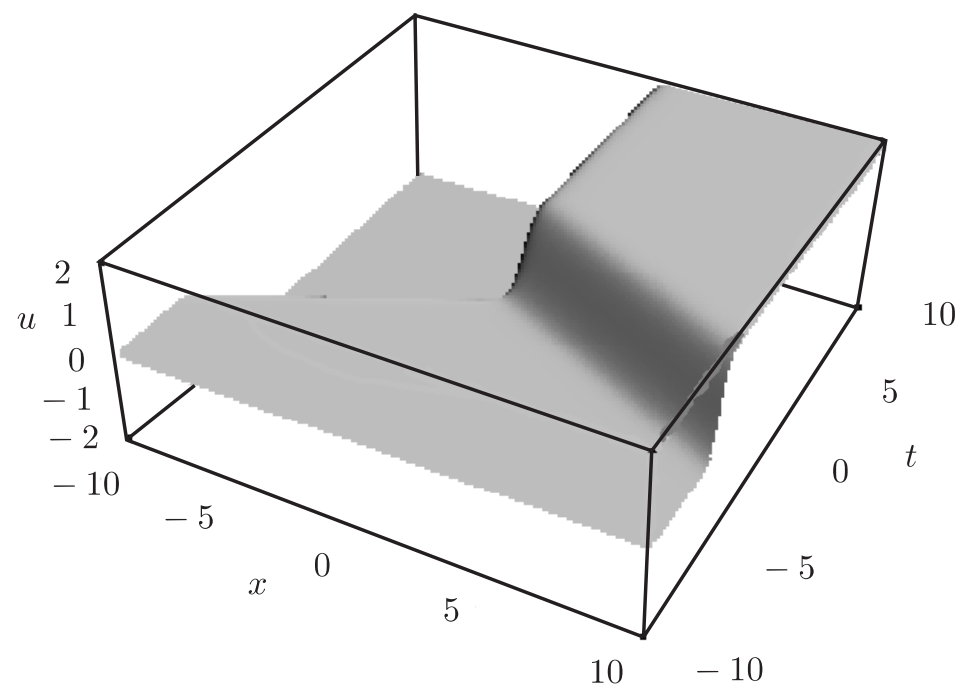

Рис. 4. Двухфронтовое решение НФ Бюргерса (уравнение (28)); $k_{1}=2, k_{2}=-2$. 
Препятствие выявляется при анализе в первом порядке. Если перенести все линейные члены $u_{x x}$ из первого порядка в НФ, т.е. взять $\mu=\alpha_{4}$, а также выбрать $u^{(1)}$ таким образом, чтобы было решено гомологическое уравнение для случая одного фронта и при этом $u^{(1)}$ было явной функцией от $x$ и $t$,

$$
\begin{gathered}
u^{(1)}=u_{r}^{(1)}(x, t)+\left(\alpha_{1}-2 \alpha_{2}-\alpha_{3}+2 \alpha_{4}\right) q u_{x}-\frac{1}{2}\left(2 \alpha_{1}-\alpha_{2}+\alpha_{3}-2 \alpha_{4}\right) u^{2}, \\
q \equiv \partial_{x}^{-1} u,
\end{gathered}
$$

то препятствие получится в своей канонической форме

$$
\begin{gathered}
R^{(1)}=\gamma_{3}^{1} R_{21}=\gamma_{3}^{1}\left(S^{(2)} G^{(1)}-S^{(1)} G^{(2)}\right)=\gamma_{3}^{1}\left(u S^{(2)}-u_{x} G^{(2)}\right) \\
\gamma_{3}^{1}=2 \alpha_{1}-\alpha_{2}-2 \alpha_{3}+\alpha_{4} .
\end{gathered}
$$

Это препятствие показано на рис. 5 , и можно видеть, что оно является конечным во всей области взаимодействия. Гомологическое уравнение теперь принимает вид

$$
\partial_{t} u_{r}^{(1)}=2 \partial_{x}\left(u u_{r}^{(1)}\right)+\partial_{x}^{2} u_{r}^{(1)}+\gamma_{3}^{1} R_{21}
$$

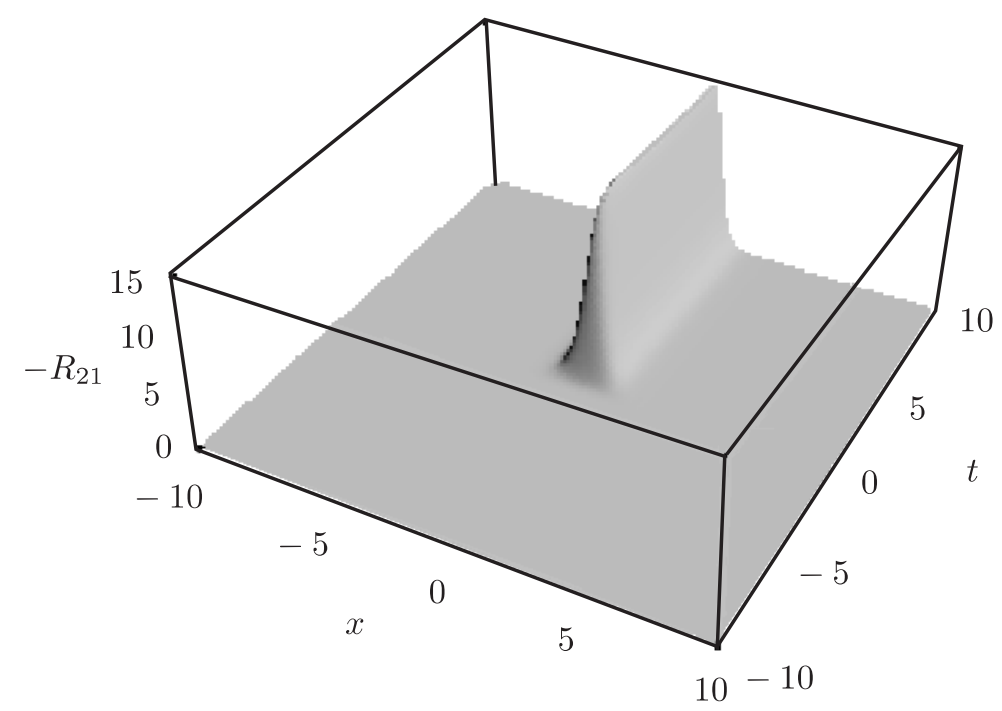

Рис. 5. Каноническое препятствие $-R_{21}$ (с противополжным знаком) для двухфронтового решения уравнения Бюргерса (уравнение (31)); параметры те же, что и на рис. 4.

Следует заметить, что из двух членов в $R_{21}$ только $u S^{(2)}$ не может быть учтен с помощью дифференциальных полиномов в ПТП. Напротив, легко видеть, что подстановка $u_{r}^{(1)}=G^{(2)}$ в однородную часть гомологического уравнения приводит к уравнению

$$
-\partial_{t} G^{(2)}+2 \partial_{x}\left(u G^{(2)}\right)+\partial_{x}^{2} G^{(2)}=2 u_{x} G^{(2)} .
$$


Как и ожидалось, численное решение гомологического уравнения демонстрирует существование секулярного члена, что указьвает на наличие симметрии внутри $R_{21}$.

Чтобы извлечь эту симметрию, используем рекуррентное соотношение между симметриями иерархии Бюргерса:

$$
S^{(n+1)}=S_{x}^{(n)}+u S^{(n)}+u_{x} G^{(n)}=S_{x}^{(n)}+R_{n 1}+2 u_{x} G^{(n)} .
$$

В частности, при $n=2$ имеем

$$
S^{(3)}=S_{x}^{(2)}+R_{21}+2 u_{x} G^{(2)} \Rightarrow R_{21}=S^{(3)}-S_{x}^{(2)}-2 u_{x} G^{(2)},
$$

и гомологическое уравнение (32) принимает вид

$$
\partial_{t} u_{r}^{(1)}=2 \partial_{x}\left(u u_{r}^{(1)}\right)+\partial_{x}^{2} u_{r}^{(1)}+\gamma_{3}^{1}\left(S^{(3)}-S_{x}^{(2)}-2 u_{x} G^{(2)}\right) .
$$

Выберем теперь $\mu$ (коэффициент при $S^{(3)}$ в НФ) в виде

$$
\mu=\alpha_{4}+\gamma_{3}^{1}=2 \alpha_{1}-\alpha_{2}-2 \alpha_{3}+2 \alpha_{4},
$$

что дает поправку к измененной волновой скорости (уравнение (29)). Далее, добавим подходящую поправку к $u_{d}^{(1)}$, с тем чтобы учесть член $-2 \gamma_{3}^{1} u_{x} G^{(2)}$ в уравнении (36), согласно уравнению (33).

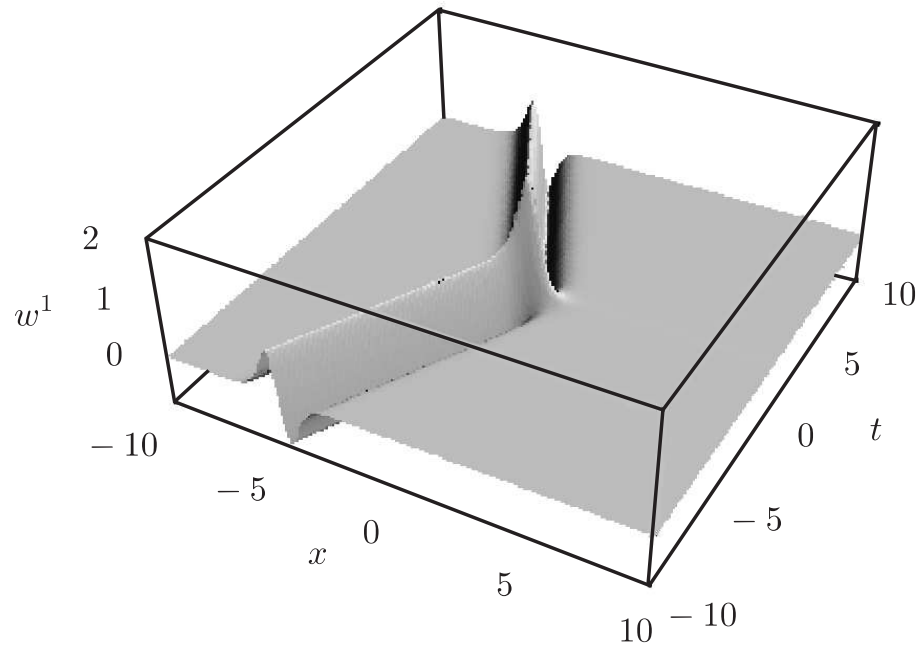

Рис. 6. Ограниченный вклад препятствия $S_{x}^{(2)}$ в $u_{r}^{(1)}$ в уравнении (38); параметры те же, что и на рис. 4.

Теперь уравнение для $u_{r}^{(1)}$ принимает вид

$$
\partial_{t} u_{r}^{(1)}=2 \partial_{x}\left(u u_{r}^{(1)}\right)+\partial_{x}^{2} u_{r}^{(1)}-\gamma_{3}^{1} S_{x}^{(2)} .
$$

Член $S_{x}^{(2)}$ не стремится асимптотически ни к какой симметрии. Численное решение уравнения (38) показывает, что появляется новая ограниченная волна (см. рис. 6).

Благодарности. Мы благодарим Г. Берде, Л. Калякина и Ю. Кодаму за полезные обсуждения. 


\section{Список литературы}

[1] D. J. Kaup. SIAM J. Appl. Math. 1976. V. 31. P. 121.

[2] D. J. Kaup, A. S. Newell. Proc. Roy. Soc. London. A. 1978. V. 361. P. 413.

[3] В.И. Карпман, Е. М. Маслов. ЖЭТФ. 1977. Т. 73. С. 537.

[4] A. C. Newell. The Inverse Scattering Transform. In: Solitons. Eds. R. K. Bullough, P. J. Caudrey. Berlin: Springer, 1980. P. 177.

[5] Л. А. Калякин. УМН. 1989. Т. 44. № 1. С. 5.

[6] Л. А. Калякин. ТМФ. 1992. Т. 92. № 1. С. 62.

[7] L. A. Kalyakin. Physica D. 1995. V. 87. P. 193.

[8] Л. А. Калякин, В. А. Лазарев. ТМФ. 1997. Т. 112. № 1. С. 92.

[9] Y. Kodama. Phys. Lett. A. 1985. V. 112. P. 193.

[10] Y. Kodama. Physica D. 1985. V. 16. P. 14.

[11] Y. Kodama. Normal Form and Solitons. In: Topics in Soliton Theory and Exactly Solvable Nonlinear Equation. Proc. of the Conf. on Nonlinear Evolutional Equations, Solitons and the Inverse Scattering Transform (Oberwolfach, Germany, July 27 - August 2, 1986). Eds. M. J. Ablowitz, B. Fuchssteiner, M. Kruskal. Singapore: World Scientific, 1987. P. 319.

[12] Y. Kodama, A. V. Mikhailov. Obstacles to asymptotic integrability. In: Algebraic Aspects of Integrable Systems. Eds. A. S. Fokas, I. M. Gelfand. Boston: Birkhauser, 1997. P. 173.

[13] Y. Hiraoka, Y. Kodama. Normal Form and Solitons. Lecture Notes, Euro Summer School 2001 (Cambridge, The Isaac Newton Institute, August 13-24, 2001) (unpublished).

[14] Y. Kodama, T. Taniuti. J. Phys. Soc. Japan. 1979. V. 47. P. 1706.

[15] T. Fokas, L. Luo. Contemp. Math. 1996. V. 200. P. 85.

[16] R. A. Kraenkel, J. G. Pereira, E. C. de Rey Neto. Phys. Rev. E. 1998. V. 58. P. 2526.

[17] R. A. Kraenkel, M. A. Manna, V. Merle, J. C. Montero, J. G. Pereira. Phys. Rev. E. 1996. V. 54. P. 2976.

[18] R. A. Kraenkel. Phys. Rev. E. 1998. V. 57. P. 4775.

[19] R. A. Kraenkel, M. Senthilvelan, A. I. Zenchuk. J. Math. Phys. 2000. V. 41. P. 3160.

[20] A. Degasperis, S. V. Manakov, P. M. Santini. Physica D. 1997. V. 100. P. 187.

[21] Y. Kodama, T. Taniuti. J. Phys. Soc. Japan. 1978. V. 45. P. 298.

[22] Y. Kodama. J. Phys. Soc. Japan. 1978. V. 45. P. 311.

[23] B. Fuchssteiner, A. S. Fokas. Physica D. 1981. V. 4. P. 47.

[24] M. J. Ablowitz, P. A. Clarkson. Solitons, Nonlinear Evolution Equations and Inverse Scattering. Cambridge: Cambridge Univ. Press, 1991.

[25] H. Tasso. J. Phys. A. 1996. V. 29. P. 7779.

[26] A.S. Fokas, R.H.J. Grimshaw, D. E. Pelinovsky. J. Math. Phys. 1996. V. 37. P. 3415.

[27] Y. Kodama. Phys. Lett. A. 1987. V. 123. P. 276.

[28] R. Hirota. Phys. Rev. Lett. 1971. V. 27. P. 1192.

[29] J. P. Keener, D. W. McLaughlin. Phys. Rev. A. 1977. V. 16. P. 777.

[30] R. L. Sachs. SIAM J. Math. Anal. 1983. V. 14. P. 674.

[31] R. L. Sachs. SIAM J. Math. Anal. 1984. V. 15. P. 468. 